\title{
Phase Time and Envelope Time in Time-distance Analysis and Acoustic Imaging
}

\author{
Dean-Yi Chou \\ Physics Department, Tsing Hua University, Hsinchu, 30043, Taiwan, R.O.C. \\ Thomas L. Duvall, Jr. \\ Laboratory for Astronomy and Solar Physics, NASA Goddard Space Flight Center, Greenbelt, MD 20771, U.S.A. \\ and \\ the TON Team ${ }^{1}$
}

\begin{abstract}
Time-distance analysis and acoustic imaging are two related techniques to probe the local properties of solar interior. In this study, we discuss the relation of phase time and envelope time between the two techniques. The location of the envelope peak of the cross correlation function in time-distance analysis is identified as the travel time of the wave packet formed by modes with the same $\omega / l$. The phase time of the cross correlation function provides information of the phase change accumulated along the wave path, including the phase change at the boundaries of the mode cavity. The acoustic signals constructed with the technique of acoustic imaging contain both phase and intensity information. The phase of constructed signals can be studied by computing the cross correlation function between time series constructed with ingoing and outgoing waves. In this study, we use the data taken with the Taiwan Oscillation Network (TON) instrument and the Michelson Doppler Imager (MDI) instrument. The analysis is carried out for the quiet Sun. We use the relation of envelope time versus distance measured in timedistance analyses to construct the acoustic signals in acoustic imaging analyses. The phase time of the cross correlation function of constructed ingoing and outgoing time series is twice the difference between the phase time and envelope time in time-distance analyses as predicted. The envelope peak of the cross correlation function between constructed ingoing and outgoing time series is located at zero time as predicted for results of one-bounce at $3 \mathrm{mHz}$ for all four data sets and two-bounce at $3 \mathrm{mHz}$ for two TON data sets. But it is different from zero for other cases. The cause of the deviation of the envelope peak from zero is not known.
\end{abstract}

\footnotetext{
${ }^{1}$ The TON Team includes: Ming-Tsung Sun (Department of Mechanical Engineering, Chang-Gung University, Kwei-San, Taiwan) and Hsiang-Kuang Chang (Physics Department, Tsing Hua University, Hsinchu, Taiwan); Antonio Jimenez and Maria Cristina RabelloSoares (Instituto Astrofisica de Canarias, Observatorio del Teide, Tenerife, Spain); Guoxiang Ai and Gwo-Ping Wang (Huairou Solar Observing Station, Beijing Observatory, Beijing, China); Philip Goode and William Marquette (Big Bear Solar Observatory, New Jersey Institute of Technology, Newark, NJ 07102, U.S.A.); Shuhrat Ehgamberdiev and Oleg Ladenkov (Ulugh Beg Astronomical Institute, Tashkent, Uzbekistan).
} 
Subject headings: Sun: oscillations - Sun: magnetic field - sunspots 


\section{Introduction}

Time-distance analysis and acoustic imaging are two related techniques to probe the local properties of the solar interior. A resonant p-mode is trapped and multiply-reflected in a cavity between the surface and a layer in the solar interior. The acoustic signal emanating from a point at the surface propagates downward to the bottom of the cavity and back to the surface at a different horizontal distance from the original point. Different p-modes have different paths and arrive at the surface with different times and different distances from that point. The modes with the same angular phase speed $\omega / l$ have approximately the same ray path, where $\omega$ is the mode frequency and $l$ is the spherical harmonic degree. In time-distance analysis, the travel time of a wave packet formed by the modes with the same $\omega / l$ versus the travel distance is determined from the cross-correlation function of signals measured at two locations (Duvall et al. 1993). The phase time of the cross-correlation can also be determined (Duvall et al. 1996). The perturbation in phase time due to local inhomogeneities can be measured and inverted to infer the flow, magnetic field, and temperature below the solar surface (Jefferies et al. 1994, D'Silva et al. 1996, Duvall et al. 1996, Kosovichev 1996, Braun 1997, Bogdan et al. 1997, Kosovichev \& Duvall 1997, more updated references?).

In acoustic imaging, the signals measured at the solar surface are coherently added, based on the timedistance relation, to construct the signal at a point on the solar surface or in the solar interior (Chang et al. 1997, Lindsey \& Braun 1997, Chen et al. 1998, Braun et al. 1998, Chou et al. 1999). For a target point on the surface, one can use the measured time-distance relation. For a target point below the surface, one has to use the time-distance relation computed with a solar standard model. The constructed signal contains information on intensity and phase. The phase of a constructed time series contains information on the phase change as the wave packet propagates from the target point to the observed point. This phase change relates to the phase time and envelope time in the time-distance analysis. In this paper, we study the relation of phase time and envelope time between time-distance analysis and acoustic imaging analysis.

The phase of the constructed time series in acoustic imaging depends on the time-distance relation used. If the travel time used to construct the signals is shifted by a constant, the constructed time series is also shifted by the same amount (Chen et al. 1998, Chou et al. 1999). In this study, we use the measured travel time (envelope time) in time-distance analysis to construct signals in acoustic imaging. The measured time-distance relation is sensitive to the frequency (Jefferies et al. 1994, D'Silva et al. 1999). To eliminate the frequency dependence, we use the same data set to measure the time-distance relation and to construct signals at the solar surface. Also, when comparing MDI and TON data, we isolate the same frequency bands for analysis.

\section{Observations and Data}

We use the helioseismic data taken with the TON instruments and the SOI/MDI instruments aboard SOHO. The TON data are full-disk K-line images recorded by a 16-bit $1080 \times 1080$ water-cooled CCD. The images are taken at a rate of one image per minute. The diameter of the Sun is about 1000 pixels. The measured amplitude of intensity oscillations is about $2.5 \%$. A discussion of the TON project and its instruments is given by Chou et al. (1995). The preliminary data reduction of the TON data, such as flatfielding and registration, is given by Chen, Chou, \& TON Team (1996). Two 512-minute time series on 1996 August 3 are used in this study. One is taken in Tenerife, defined as TON-1; another is taken in Tashkent, defined as TON-2. The overlap of the two time series is 172 minutes.

The SOI/MDI experiment generates Dopplergrams, magnetograms and continuum images at different scales. A summary of the SOI/MDI project was given by Scherrer (1995). The MDI data used in this study are $1024 \times 1024$ full-disk Doppler images with one minute cadence. We use two 1024-minute time series on July 6 of 1996 (defined as MDI-1) and July 11 of 1996 (defined as MDI-2). (Dean: I only used the first 512 minutes for the time-distance analysis)

Each observed full-disk image is transformed into $\sin \theta-\phi$ coordinates with the GRASP package developed by GONG, where $\theta$ and $\phi$ are the latitude and the longitude, respectively, in a spherical coordinate system aligned along the solar rotation axis. The solar differential rotation at the surface is removed with an observed surface differential rotation velocity (Libbrecht \& Morrow 1991). The data is filtered with a Gaussian filter of FWHM $=1 \mathrm{mHz}$ centered at 3 and $4 \mathrm{mHz}$. Details of data preparation are discussed in Chou et al. (1999). 


\section{Phase Time and Envelope Time in Time- distance Analyses}

The cross-correlation function $C(\tau, \Delta)$ between a central point and an annular region at an angular distance of $\Delta$ is computed with

$$
C(\tau, \Delta)=\int_{0}^{T} f(t) \cdot f(t+\tau, \Delta) d t
$$

where $f(t)$ is the signal measured at the central point, and $f(t+\tau, \Delta)$ is the signal averaged over an annular region at an angular distance of $\Delta$ from the central point. The width of the annular region is $0.35^{\circ}$ for the TON data and $0.12^{\circ}$ for the MDI data. This procedure is repeated for different central points in a region of $87^{\circ} \times 75^{\circ}$ at the disk center. The average cross-correlation function $\bar{C}(\tau, \Delta)$ is obtained by averaging over this region. For a fixed angular distance $\Delta$, $\bar{C}(r, \Delta)$ has the approximate form of a Gabor wavelet,

$$
G(\tau)=A \cos \left(2 \pi \nu\left[\tau-\tau_{p h}\right]\right) \cdot \exp \left(-\frac{\left[\tau-\tau_{e n}\right]^{2}}{2 \sigma^{2}}\right),
$$

where $A, \sigma$ and $\nu$ are the amplitude, width and frequency respectively. The location of the peak of the envelope, $\tau_{e n}$ (envelope time), is identified as the travel time of the wave packet. The phase time, $\tau_{p h}$, is undetermined to multiples of the period, $\nu^{-1}$. The measured average cross-correlation function at a fixed $\Delta, \bar{C}(\tau, \Delta)$, is fitted to the Gabor wavelet in equation (2) to obtain the envelope time $\tau_{e n}$ and the phase time $\tau_{p h}$. This procedure is repeated for different $\Delta$ to obtain the time-distance relations, $\tau_{e n}$ versus $\Delta$ and $\tau_{p h}$ versus $\Delta$.

The above analysis is carried out for two TON data sets (TON-1 and TON-2) and one MDI data set (MDI-1). The range of $\Delta$ is $1.75-24.85^{\circ}$ for TON data and $1.08-24.96^{\circ}$ for MDI data. The measured time-distance relations (both $\tau_{e n}$ and $\tau_{p h}$ ) from the three data sets are very close. Examples of measured time-distance relations from the TON-1 and MDI-1 data sets at $3 \mathrm{mHz}$ are shown in Figures 1 and 2, respectively. The upper solid curve is the phase time $\tau_{p h}$; and the lower solid curve is the envelope time $\tau_{e n}$. Usually $\tau_{p h}$ can be determined more accurately than $\tau_{e n}$. The measured $\tau_{p h}$ versus angular distance is rather smooth and can be fitted well with a fifthorder polynomial. The fluctuation of measured $\tau_{e n}$ is large, and it is difficult to be sure that the fit is good. In this study we will use the relation of $\tau_{e n}$ versus angular distance to construct acoustic signals in acoustic imaging. Thus we have to find a better way to measure $\tau_{e n}$.

The difference between $\tau_{p h}$ and $\tau_{e n}$ increases slowly with angular distance. The increase is approximately linear. An example (TON-1 at $3 \mathrm{mHz}$ ) is shown in Figure 3 . The large fluctuation is caused by the inaccuracy in determining $\tau_{e n}$. The dashed line is a linear fit. The results from the other two data sets also have this property: $\tau_{p h}-\tau_{e n}$ increase slowly and linearly with angular distance. Since measured $\tau_{p h}-\tau_{e n}$ can be fitted well by a linear function, we subtract this linear function from the fitted $\tau_{p h}$ to obtain $\tau_{e n}$, which corresponds to the dashed lines in Figures 1 and 2. It is noted that the value of $\tau_{p h}-\tau_{e n}$ is strongly dependent on the frequency. But it always increases slowly and approximately linearly with angular distance.

The modes with the same $\omega / l$ have approximately the same ray path, and form a wave packet. If the power distribution of modes is sharply peaked around some value $\omega_{0}$ and the second derivative of $\vec{k}$ with respect to $\omega$ is small, with the expression for wavefunctions in D'Silva (1996) and the technique in Jackson (1975), the wavefunction of the wave packet can be expressed as

$$
\Psi(\vec{r}, t) \approx e^{i\left(\omega_{0} T\left(\vec{r}_{0}, \vec{r}\right)-\int_{r_{0}}^{*} \vec{k}_{0} \cdot d \vec{s}-\alpha\right)} \Psi\left(\vec{r}_{0}, t-T\left(\vec{r}_{0}, \vec{r}\right)\right),
$$

where $\left.T\left(\vec{r}_{0}, \vec{r}\right) \equiv \int_{\vec{r}_{0}}^{\vec{r}}(\partial \vec{k} / \partial \omega)\right|_{0} \cdot d \vec{s}$ is the travel time of the wave packet from $\vec{r}_{0}$ to $\vec{r}$ along the ray path. The term $\int_{\vec{r}_{0}}^{\vec{r}} \vec{k}_{0} \cdot d \vec{s}$ is the phase change accumulated along the wave path from $\vec{r}_{0}$ to $\vec{r}$. The additional phase $\alpha$ accounts for the phase change at the boundaries of mode cavity. Equation (3) shows that, apart from an overall phase factor, the wave packet approximately maintains its form.

The cross-correlation function of signals at $\vec{r}_{0}$ and $\vec{r}$ is computed as equation (1)

$$
\begin{aligned}
& C\left(\tau, \vec{r}_{0}, \vec{r}\right) \\
= & \int \Psi\left(\vec{r}_{0}, t\right) \cdot \Psi^{*}(\vec{r}, t+\tau) d t \\
\approx & e^{-i\left(\omega_{0} T-\int_{p_{0}}^{*} \vec{k}_{0} \cdot d \vec{s}-\alpha\right)} \int \Psi\left(\vec{r}_{0}, t\right) \cdot \Psi^{*}\left(\vec{r}_{0}, t+\tau-T\right) d t \\
= & e^{-i\left(\omega_{0} T-\int_{p_{0}}^{*} \vec{k}_{0} \cdot d \vec{s}-\alpha\right)} \tilde{C}\left(\tau-T, \vec{r}_{0}\right)
\end{aligned}
$$

where $\tilde{C}\left(\tau-T, \vec{r}_{0}\right)$ is the auto-correlation function of the signal at $\vec{r}_{0}$, which is peaked at $\tau=T$. Thus the envelope of $C\left(\tau, \vec{r}_{0}, \vec{r}\right)$ is peaked at $\tau=T$. The difference between envelope time and phase time of 


$$
C\left(\tau, \vec{r}_{0}, \vec{r}\right) \text { is }
$$

$$
\begin{aligned}
\tau_{p h}-\tau_{e n} & =\int_{\vec{r}_{0}}^{\vec{r}} \frac{\vec{k}_{0}}{\omega_{0}} \cdot d \vec{s}-T+\frac{\alpha}{\omega_{0}} \\
& =\int_{\vec{r}_{0}}^{\vec{r}} \frac{\hat{v}_{p}}{v_{p}} \cdot d \vec{s}-\int_{\vec{r}_{0}}^{\vec{r}} \frac{\hat{v}_{g}}{v_{g}} \cdot d \vec{s}+\frac{\alpha}{\omega_{0}}
\end{aligned}
$$

where $\hat{v}_{p}$ and $\hat{v}_{g}$ are the unit vectors of phase velocity and group velocity, respectively. The difference between the phase velocity and the group velocity is small except near the solar surface.

\section{Phase Time and Envelope Time in Acous- tic Imaging}

The acoustic signals at a point on the solar surface or in the solar interior can be constructed with the technique of acoustic imaging (Chang et al. 1997, Chou et al. 1999). Since the goal of this study is to compare phase information in time-distance analysis and acoustic imaging, we will use the measured time-distance relation to construct acoustic signals at the solar surface. To study the phase information of constructed time series, we compare the time series constructed with outgoing waves and the time series constructed with ingoing waves by computing the cross correlation function of two time series (Chen et al. 1998). The outgoing time series is constructed with the ordinary time-distance curve; and the ingoing time series is constructed with the time-reversed time-distance curve. Since our data is filtered with a Gaussian filter, the cross correlation function has approximately the form of a Gabor wavelet as in equation (2). The phase time $\tau_{p h}^{\prime}$ and envelope time $\tau_{e n}^{\prime}$ of the cross correlation function can be be determined by fitting it with the Gabor wavelet. Here we use the superscript prime to distinguish from the phase time and envelope time in the time-distance analysis. The phase time $\tau_{p h}^{\prime}$ is also undetermined to multiples of the modulation period.

In this study, we use the envelope time measured in time-distance analysis to reconstruct acoustic signals. The envelope time measured in time-distance analysis is interpreted as the travel time of a wave packet. Thus $T=\tau_{e n}$ in equation (3). To construct the signal at $\vec{r}_{0}$ and $t=0$, we collect signals measured at $\vec{r}$ and $t=T$, which is $\Psi(\vec{r}, T)$, for various $\vec{r}$ and $T$, where $T$ and $\left|\vec{r}-\vec{r}_{0}\right|$ satisfies the time-distance relation. ¿From equation (3), we have

$$
\Psi(\vec{r}, T) \approx e^{i\left(\omega_{0} T\left(\vec{r}_{0}, \vec{r}\right)-\int_{\rho_{0}}^{r} \vec{k}_{0} \cdot d \vec{s}-\alpha\right)} \Psi\left(\vec{r}_{0}, 0\right)
$$

$$
=e^{-i \omega_{0}\left(r_{p h}-\tau_{s n}\right)} \Psi\left(\vec{r}_{0}, 0\right) .
$$

Equation (6) shows that the collected signals differ from the original signal only by a phase factor $\exp \left(-i \omega_{0}\left(\tau_{p h}-\tau_{e n}\right)\right)$ This phase varies slightly with distance. If the range of distance in the coherent sum is not large, the variation of this phase with distance is negligible.

Based on the above discussion, we have two predictions about the cross-correlation function of constructed ingoing and outgoing time series, if we use the envelope time $\tau_{e n}$ measured in time-distance analysis to construct signals in acoustic imaging. First, the envelope time of the cross-correlation $\tau_{e n}^{\prime}$ is zero. Second, the phase time of the cross-correlation $\tau_{p h}^{\prime}$ is twice $\tau_{p h}-\tau_{e n}$. This is because the phases of constructed outgoing time series and ingoing time series are different from the phase of the original time series by $\tau_{p h}-\tau_{e n}$ and $-\left(\tau_{p h}-\tau_{e n}\right)$, respectively. We will discuss the comparison of these two predictions with observations.

We have analyzed two TON data sets (TON-1 and TON-2). For each data set, we use the time-distance relation measured from this data set to construct the signals. We have also analyzed two MDI data sets (MDI-1 and MDI-2). But we use the time-distance relation measured from MDI- 1 to construct the signals for MDI-1 and MDI-2. The range of time-distance curves used to construct signals is $3.4-7.5^{\circ}$ for TON data, and $1.8-5.5^{\circ}$ for MDI data. The target region is a quiet region near the disk center. Its size is $17.6 \times 11.7^{\circ}$ for TON data and $12.0 \times 12.0^{\circ}$ for MDI data. The regions used to construct signals are also in the quiet Sun.

In this study, we use two different ways to determine $\tau_{e n}^{\prime}$ and $\tau_{p h}^{\prime}$. In the first way, we determine the parameters of the cross-correlation function of constructed ingoing and outgoing time series at each point by fitting it to a Gabor wavelet, and then average the parameters over the target region. In the second way, we average the cross-correlation function over the target region, and then determine the parameters by fitting the average cross-correlation to a $\mathrm{Ga}$ bor wavelet. The two ways yield very close results. In the tables, we give only the result from the first way. The parameters of the cross-correlation function from the four different data sets are shown in Tables 1 and 2 , which correspond to $3 \mathrm{mHz}$ and $4 \mathrm{mHz}$, respectively. The third column of the tables is the amplitude $A$ of the envelope of the cross correlation function. The 
fourth column is the modulation period, $\nu^{-1}$. The fifth and sixth columns are the envelope time $\tau_{e n}^{\prime}$ and the phase time $\tau_{p h}^{\prime}$ of the cross correlation function, respectively. The last column is the predicted phase time, which equals to $2 N_{s}\left(\tau_{p h}-\tau_{e n}\right)$, where $N_{s}$ is the number of the skip (bounce). The value of $\tau_{p h}-\tau_{e n}$ quoted here is the average over the range of angular distance used to construct signals.

The acoustic signals can be constructed with the signals after more than one bounce from the target point. The time-distance curve of multiple bounces used in this study is obtained by multiplying both measured travel time and distance of one-bounce by the number of bounces. The results of 2-bounce and 3 -bounce are also shown in tables 1 and 2 .

The value of $\tau_{e n}^{\prime}$ is close to zero for 1-bounce results of all four data sets and 2-bounce results of TON-1 and TON-2 at $3 \mathrm{mHz}$ as predicted. But it is not close to zero for the other cases. We will discuss the possible causes for the large $\tau_{e n}^{\prime}$ in these cases. On the other hand, the measured $\tau_{p h}^{\prime}$ is close to the predicted value for all cases we have studied, except one case (2-bounce, TON-2 at $3 \mathrm{mHz}$ ). We conclude that the measured $\tau_{p h}^{\prime}$ is in good agreement with the predicted value.

From equation (3), the constructed time series depends on the time-distance curve used in acoustic imaging. If the travel time used is shifted by a constant $\Delta t$, the constructed outgoing time series will shift by $\Delta t$, and the constructed ingoing time series by $-\Delta t$. The cross correlation function of ingoing and outgoing time series will shift by $-2 \Delta t$. It leads to that both phase time and envelope of the cross correlation function shift by $-2 \Delta t$. Therefore, it is unlikely that the deviation of measured $\tau_{e n}^{\prime}$ from the predicted value is caused by the inaccuracy of measured envelope time $\tau_{e n}$ used in acoustic imaging, because measured $\tau_{p h}^{\prime}$ is rather close to the predicted value.

The determination of $\tau_{e n}^{\prime}$ in the fit is less accurate than $\tau_{p h}^{\prime}$ and it is difficult to estimate the error of $\tau_{e n}^{\prime}$. But it is unlikely that the error in the fit is large as the value of measured $\tau_{e n}^{\prime}$, such as 2 minutes, in some cases, though we can not totally rule out this possibility.

The power spectra of filtered data are not exactly a Gaussian, though we use a Gaussian filter (D'Silva et al. 1999). The power spectra of constructed signals have a further distortion from a Gaussian because different frequencies have different lifetimes. Thus the envelope of the cross-correlation function of constructed outgoing and ingoing time series is not exactly a Gaussian. Fitting the cross-correlation function to a Gabor wavelet may cause some error in determining $\tau_{e n}^{\prime}$. But we believe this error can not account for the large value of measured $\tau_{e n}^{\prime}$ alone.

\section{Discussion}

This study confirms that the phase time $\tau_{p h}^{\prime}$ of the cross-correlation function of constructed ingoing and outgoing time series in acoustic imaging is equal to twice the difference between phase time $\tau_{p h}$ and envelope time $\tau_{e n}$ in time-distance analyses. The difference between phase time and envelope time contains two information: 1) the difference between the phase velocity and the group velocity and 2) the phase change at the boundaries of mode cavity.

If the target point is located inside a sunspot, the presence of magnetic field would modify both the phase time and the envelope time. First, the presence of magnetic field would modify the dispersion relation of waves and would change the difference between the phase velocity and the group velocity. Second, the magnetic field would modify the depth of the upper boundary of mode cavity. It makes both envelope time and phase time change. Third, the magnetic field would also modify the physical conditions at the upper boundary of mode cavity. It would modify of the phase change as the wave packet reflected at the boundary. Fourth, the phase of a wave packet would change as it goes across lateral boundaries of the sunspot. Fifth, the flow caused by the presense of the sunspot would change both enevlope time and phase time. But the changes in envelope time and phase time are equal. Therefore, the comparison of deviations of measured $\tau_{p h}^{\prime}$ and $\tau_{e n}^{\prime}$ inside a sunspot from the values in the quiet Sun provides information on the structure of the sunspot.

We are grateful to the GONG Data Team for providing the software package GRASP. DYC and the TON project were supported by NSC of ROC under grants NSC-87-2112-M-007-044. TLD and the MDI project were supported by the SOI-MDI contract NAG5-3077 at Stanford University.

\section{REFERENCES}

Bogdan, T. J., Braun, D. C., Lite, B. W., \& Thomas, 
J. H., 1997, ApJ, 492, 379

Braun, D. C., Lindsey, C., Fan, Y., \& Fagan, M., 1992, ApJ, 502, 968

Braun, D. C., 1997, ApJ, 487, 447

Chang, H.-K., Chou, D.-Y., LaBonte, B., \& the TON Team, 1997, Nature, 389, 825 (Paper I)

Chen, K.-R., Chou, D.-Y., \& the TON Team, 1996, ApJ, 465, 985

Chen, H.-R., Chou, D.-Y., Chang, H.-K., Sun, M.-T., Yeh, S.-J, LaBonte, B., \& the TON Team, 1998, ApJ, 501, L139

Chou, D.-Y., Sun, M.-T., Huang, T.-Y. et al., 1995, Sol. Phys., 160, 237

Chou, D.-Y., Chang, H.-K., Sun, M.-T., LaBonte, B., Chen, H.-R., Yeh, S.-J., and the TON Team, 1999, ApJ, 514, 979

D'Silva, 1996, ApJ, 469, 964

D'Silva, S., Duvall, T. L. Jr., Jefferies, S. M., \& Harvey, J. W., 1996, ApJ, 471, 1030

D’Silva, S., Kuhn, J. R., Jefferies, S. M., Harvey, J. W., \& Duvall, T. L. Jr., 1999, ApJ, in press

Duvall, T. L. Jr., Jefferies, S. M., Harvey, J. W., \& Pomoerantz, M. A., 1993, Nature, 362430 ,

Duvall, T. L. Jr., D'Silva, S., Jefferies, S. M., Harvey, J. W., and \& Schou, J., 1996, Nature, 379, 235.

Duvall, T. L. Jr., 1998, private communication.

Jackson, J. D., 1975, Classical Electrodynamics (New York: Wiley), p.301

Jefferies, S. M., Osaki, Y., Shibahashi, H., Duvall, T. L. Jr., Harvey, J. W., \& Pomoerantz, M. A., 1994, ApJ, 434 795,

Kosovichev, A. G., 1996, ApJ, 461, L55

Kosovichev, A. G. \& Duvall, T. L. Jr., 1997, in Solar Convection and Oscillations and Their Relationship, ed. J. Christensen-Dalsgaard and F. Pijpers (Dordrecht: Kluwer), 241

Libbrecht, K. G. \& Morrow, C. A., 1991, in Solar Interior and Atmosphere, ed. A. N. Cox et al. (Tucson: University of Arizona Press), p.479
Lindsey, C. \& Braun, D. C., 1997, ApJ, 485, 895

Scherrer, P. H. et al., 1995, Sol. Phys., 162, 129 
TABLE 1

Phase time and envelope time in acoustic imaging (3MHz).

\begin{tabular}{ccccccc}
\hline \hline data & no. of skip & amplitude & period $(\min )$ & $\tau_{e n}^{\prime a}$ & $\tau_{p h}^{\prime}{ }^{b}$ & predicted $\tau_{p h}^{\prime}{ }^{c}$ \\
\hline TON-1 & 1 & 0.55 & 5.27 & -0.05 & 0.75 & 0.80 \\
TON-1 & 2 & 0.57 & 5.29 & 0.05 & 1.62 & 1.60 \\
TON-1 & 3 & 0.56 & 5.33 & 0.14 & 2.45 & 2.41 \\
& & & & & & \\
TON-2 & 1 & 0.51 & 5.27 & -0.07 & 0.77 & 0.82 \\
TON-2 & 2 & 0.55 & 5.27 & 0.02 & 1.67 & 1.64 \\
TON-2 & 3 & 0.55 & 5.31 & 0.11 & 2.53 & 2.46 \\
& & & & & & \\
MDI-1 & 1 & 0.72 & 5.45 & 0.01 & 0.81 & 0.83 \\
MDI-1 & 2 & 0.67 & 5.51 & 0.47 & 1.69 & 1.67 \\
MDI-1 & 3 & 0.57 & 5.58 & 0.22 & 2.53 & 2.50 \\
& & & & & & \\
MDI-2 & 1 & 0.73 & 5.46 & 0.03 & 0.81 & 0.83 \\
MDI-2 & 2 & 0.68 & 5.53 & 0.48 & 1.69 & 1.67 \\
MDI-2 & 3 & 0.59 & 5.60 & 0.23 & 2.54 & 2.50 \\
\hline
\end{tabular}

aenvelope time, in units of the period

${ }^{b}$ phase time, in units of the period

${ }^{c}$ predicted phase time, in units of the period 
TABLE 2

Phase time and envelope time in acoustic imaging (4MHz).

\begin{tabular}{ccccccc}
\hline \hline data & no. of skip & amplitude & period $(\min )$ & $\tau_{e n}^{\prime}{ }^{\mathrm{a}}$ & $\tau_{p h}^{\prime}{ }^{\mathrm{b}}$ & predicted $\tau_{p h}^{\prime}{ }^{\mathrm{c}}$ \\
\hline TON-1 & 1 & 0.46 & 4.50 & 0.22 & 1.52 & 1.53 \\
TON-1 & 2 & 0.40 & 4.55 & 0.49 & 3.19 & 3.07 \\
TON-1 & 3 & 0.41 & 4.50 & 0.30 & 4.67 & 4.60 \\
& & & & & & \\
TON-2 & 1 & 0.42 & 4.52 & 0.21 & 1.47 & 1.49 \\
TON-2 & 2 & 0.41 & 4.57 & 0.50 & 2.35 & 2.98 \\
TON-2 & 3 & 0.42 & 4.54 & 0.36 & 4.57 & 4.48 \\
& & & & & & \\
MDI-1 & 1 & 0.64 & 4.46 & 0.14 & 1.36 & 1.36 \\
MDI-1 & 2 & 0.49 & 4.56 & 0.28 & 2.76 & 2.73 \\
MDI-1 & 3 & 0.38 & 4.55 & 0.20 & 4.18 & 4.09 \\
& & & & & & \\
MDI-2 & 1 & 0.64 & 4.46 & 0.15 & 1.36 & 1.36 \\
MDI-2 & 2 & 0.49 & 4.56 & 0.33 & 2.77 & 2.73 \\
MDI-2 & 3 & 0.39 & 4.54 & 0.28 & 4.19 & 4.09 \\
\hline
\end{tabular}

aenvelope time, in unit of period

${ }^{b}$ phase time, in unit of period

${ }^{c}$ predicted phase time, in unit of period 
Fig. 1.- Phase time and envelope time of crosscorrelation function versus angular distance measured from the TON-1 data set at $3 \mathrm{mHz}$. The upper solid curve is the phase time $\tau_{p h}$, and the lower solid curve is the envelope time $\tau_{e n}$. The dashed curve is a fit to the measured envelope time with a method described in the text.

Fig. 2.- Same as Figure 1, but measured from the MDI-1 data set.
Fig. 3.-- Difference between phase time and envelope time versus angular distance measured from the TON-1 data set at $3 \mathrm{mHz}$. The dashed line is a linear fit. 